\title{
Effective Virtual Environments
}

\section{Dr. Abdulrazaq Alsuhail Almutair}

Information \& Computer Center the Public Authority for Applied Education and Training, the Ministry of Education

State of Kuwait

Az.alsuhail@paaet.edu.kw

\begin{abstract}
Since the power of technology is in a state of continuous growth, many organisations are adopting the use of a virtual environment. There is no doubt that COVID-19 has played a significant role in accelerating and spreading the adoption of such a trend in different sectors, such as education, sales, software development, and medical sectors. Although the increasing trend may be towards building virtual teams, there are still some aspects not ideally handled by virtual environments. This paper presents the challenges and barriers, where current technologies used in establishing virtual environments have not yet been covered. In addition, it provides guidelines that can be used in designing and building software tools that support collaboration in virtual environments.
\end{abstract}

Keywords- Virtual teams, remote working, virtual workplace, hollogram

\section{INTRODUCTION}

Virtual teams (i.e. geographically dispersed collaborations that connect and collaborate using technology) have a range of possible productivity benefits. Collaboration in virtual teams applies to synchronous and asynchronous meetings and activities to accomplish shared objectives, similar to collaboration in co-located teams. Organisations may use virtual teams to enlist key experts, regardless of their physical location [1, 2]. This enables businesses to maximise their teams by using only the best talent available [3, 4]. More than $85 \%$ of working professionals were said to be part of a virtual team by 2016 [5]. Virtual teams, including co-located teams, engage in a number of collaborative activities, including formal and informal meetings through video conferencing, text, application sharing, and file transfer. As a result, virtual teams face collaboration challenges that make it difficult for them to achieve the same level of success as co-located teams $[6,1,7]$. Thus, it is critical to build technologies that can help serve virtual teams, reducing the need for expensive re-locations and alleviating the issues that occur when relocation is not an option.

Although there were some studies exploring the complexities of teamwork and the use of technology in particular contexts, such as distributed software creation, not much work has been done to examine the challenges faced by all virtual teams and their use of technology to overcome issues. This knowledge is essential for the creation and use of technology to support virtual teams.

The objectives of this paper are to address the following: (1) the factors and obstacles that obstruct virtual team collaboration, and (2) changes that can be added to the current technologies in order to better support virtual environments.

The following section starts by discussing the approach that will be used to investigate obstacles and challenges in virtual workplaces. It then details the findings of applying the approach. The third section goes through what needs to beconsidered 
when establishing developing technologies that will be used to facilitate collaboration in virtual environments.

\section{Obstacles AND Challenges IN VIRTUAL WORKPLACE}

\section{A. Approach}

In order to investigate the obstacles and challenges in a virtual environment, it was essential to understand and analyse the experience of persons involved in such environments. Therefore, a number of interviews were conducted to achieve that. The interviews carried out were based on a designed questionnaire.

The interviews were carried out in three different sectors: the software development industry, online education, and estate agencies. It was decided to do so to understand issues from different perspectives. In addition, not all people interviewed came from the same background.

\section{B. Questionnaire}

How difficult was the transition to a virtual environment?

Could you describe the team structure?

Is there any interaction with persons outside the team?

What synchronous activities are involved in your virtual workplace?

What asynchronous activities are involved in your virtual workplace?

Which activities involved in a physical workplace cannot be carried out in a virtual environment?

What software tools are used to facilitate collaboration between team members?

What areas do you think the current technologies used in your virtual workplace do not address or handle properly?

Table I: Questionnaire used in the conducted interviews
The questionnaire shown in Table I has been designed to cover the following areas:

- The transition from physical to virtual environment.

- Activities involved in virtual workplaces.

- Stakeholders involved in activities.

The next section goes through the findings in each of those area.

\section{Interview Findings}

\section{- The transition from physical to virtual environment}

It has been found that the transition was very quick and simple for software development teams and estate agencies. However, this does not give a real indication about the transition process, as most of tools needed were already installed and used. In addition, software team members come from a technical background, so they have the capabilities and skills to easily overcome technical issues without much guidance. On the other side, the transition was a complex process for the teaching and learning teams. They have to establish a system that facilitates communication with students, uploads learning materials, and homework submissions. Moreover, they need to educate their students in how to use the system.

\section{- Activities involved in virtual workplace}

Teams from different sectors need to use audio/video/text as an alternative for face-to-face conversations. Screen-sharing is also required when demonstrating slides/diagrams/artifacts. It has also been found that allocating space for resources is a useful feature for all teams. The area where all teams from different sectors agreed was not handled effectively is when it comes to drawing diagrams where it requires interactions from both parties involved in a conversation. They also added that demonstrating a physical object or model is not as effective as in a physical workplace.

\section{- Stakeholders involved in activities}

One of the challenges highlighted by all teams from different sectors was that it was not easy to get a stakeholder outside the team (e.g. client, student) set up and engaged quickly and simply. Such a complexity can lead to financial losses. 
Another issue highlighted by teacher and team leaders was the lack of eye contact with others when delivering training or virtual classes, as the current technology is not good in this aspect.

\section{RECOMMENDATIONS AND OPPORTUNITIES}

\section{A. Recommendations}

The findings obtained from the conducted interviews have been analysed to present some recommendations that may help organisations to build or move to virtual workplaces. The diagram below highlights what to consider to build an effective virtual workplace.

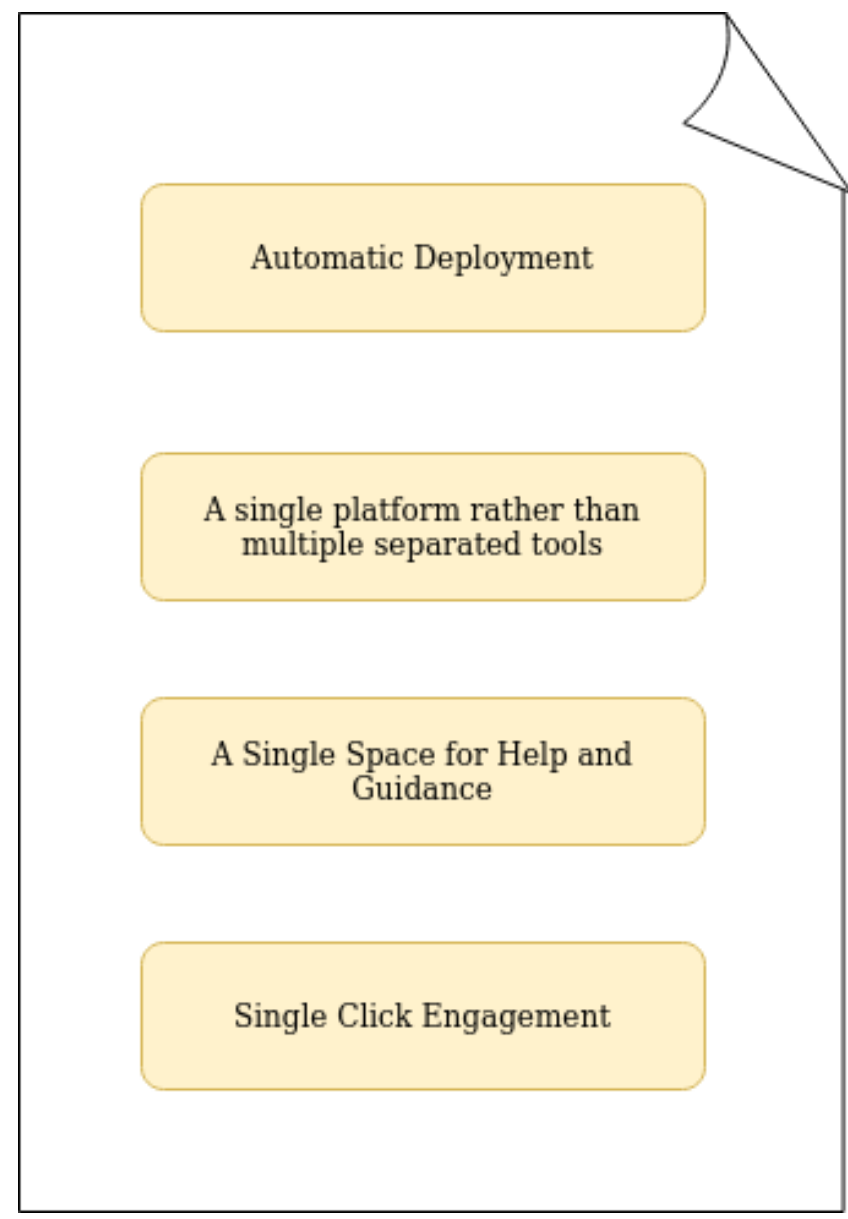

Figure 1: Recommendations suggested to build an effective virtual workplace.

\section{Automatic deployment:}

Automating the deployment of all software tools needed to carry out tasks and activities in a virtual environment makes the transition very smooth and less time-consuming compared to manual deployment, which depends very much on the user's skills and technical background. In addition, it makes the induction process for new recruits quick and simple. Moreover, it participates in mitigating security threats that may result from incorrect manual deployment.

\section{A single platform rather than multiple tools:}

Using a single platform that facilitates communication, uploads/downloads resources, and provides notifications about tasks/events/meetings, rather than multiple separate tools, has a significant impact in getting team members effectively engaged with activities happening in a virtual workspace.

\section{A single space for help and guidance:}

Providing a centralised single space for help and guidance resources in an organised and user friendly way will have the contribution of paving the way to get team members smoothly engaged. This is rather than dedicating a team to answer questions about how to use new tools and troubleshooting common issues.

\section{Single click engagement:}

Stakeholders not belonging to organisations may decide to opt out from participating in an activity if it is complicated to achieve. Involving such a stakeholder to participate in an activity (e.g. demonstrating a new property to a potential buyer) needs to be achieved with a minimum number of steps from such a stakeholder. For example, it is better to send a direct link to join the conversation rather than asking a stakeholder to download and install software.

\section{B. Opportunities}

The existing technologies used in virtual environments handle audio, video, and text communication. However, it is still not as effective as having a conversation in a physical workspace. Hologram is one of the techniques, which has not been widely used in this context. Shama (2016) described holographic technology as follows:

"A hologram is the photographic recording of light, rather than the image formation by lens, and it is used to display a fully three-dimensional image of the subject, which is seen without the aid of special glasses or other optics."

Employing such technology in this context will allow users to have a conversation with a life-sized hologram of other team members. In addition, it 
will revolutionise the way of demonstrating physical products or educational models (e.g. human body models) in online learning.

\section{CONCLUSION AND FUTURE WORK}

This paper aimed to investigate challenges and barriers experienced by persons working in a virtual workplace to enable them to effectively perform work activities. The study focused only on the technology side. Interviews were conducted in three different sectors: software industry, online learning, and estate agencies. The outcome of those interviews provided some recommendations that will contribute to building an effective virtual workspace. In addition to these recommendations, there is a great opportunity to employ holographic technology in this context.

It is planned to carry out more interviews with persons working in different industries than those covered in this paper to get more views and to explore different issues experienced in virtual environments.

\section{REFERENCES}

[1] Hinds P, Kiesler S (2002) Distributed work. MIT Press, Cambridge

[2] Lipnack J, Stamps J (1997) Virtual teams: reaching across space, time, and organizations with technology. Wiley, New York

[3] Duarte DL, Snyder NT (2006) Mastering virtual teams: strategies, tools, and techniques that succeed. Wiley, Berlin

[4] Kirkman BL, Rosen B, Tesluk PE, Gibson CB (2004) The impact of team empowerment on virtual team performance: the moderating role of face-to-face interaction. Acad Manag J 47(2):175192

[5] Solomon C (2016) Trends in global virtual teams. [Accessed on: 27/03/2021, URL: https://www.rw-3.com/resource-center/2016-

survey-report-trends-in-global-virtual-teams]

[6] Dubé L, Robey D (2009) Surviving the paradoxes of virtual teamwork. ISJ 19(1):3-30

[7] Olson GM, Olson JS (2000) Distance matters. Hum Computer Interact 15(2):139-178

[8] Shama N, Ali K (2016) A Review Paper on Holographic Technology Three-Dimensional
Visualization, International Journal of Engineering Research \& Technology, ISSN: $\underline{2278-0181}$ 